\title{
Application of Computer Technology in Optimal Design of Overall Structure of Special Machinery
}

\author{
Caiping Guo 1 \\ School of Mechanical and Electrical Engineering, Jiaozuo University, Jiaozuo 454003, Henan, China \\ Correspondence should be addressed to Caiping Guo; guocaiping@jzu.edu.cn
}

Received 24 December 2020; Revised 26 January 2021; Accepted 6 February 2021; Published 23 February 2021

Academic Editor: Ming Bao Cheng

Copyright (c 2021 Caiping Guo. This is an open access article distributed under the Creative Commons Attribution License, which permits unrestricted use, distribution, and reproduction in any medium, provided the original work is properly cited.

\begin{abstract}
With the transformation and upgrading of my country's industrial structure, the level of manufacturing automation has gradually improved. According to research, the design of mechanical products is mostly completed by improvement or innovation on the basis of existing design knowledge. Knowledge reuse is a technique to ensure the maximization of design resource utilization by reusing design knowledge. This article applies knowledge reuse technology to the development and design of mechanical products. By integrating the technical logic of the functional analysis system with the development of quality functions, the transformation of customer demand information and product function design is realized, and the task of the product design plan analysis phase is completed. This paper uses the finite element analysis software ANSYS to explore a new nonlinear finite element modeling method and conducts simulation experiments. At the same time, this paper improves the genetic algorithm, which effectively improves the optimization efficiency and completes the parameter optimization under multiobjective and multistructure conditions. From the experimental results, it takes 328.64 seconds for the basic genetic algorithm to search for the optimal solution of the complex problem. The improved time is shortened to 86.31 seconds, and the efficiency is increased by $73.74 \%$. This shows that the improved genetic algorithm has better robustness and can find the optimal solution in a shorter calculation time. For complex problems such as the optimization of the overall structure of special machinery, the improved genetic algorithm obviously helps to improve the optimization efficiency and improves the effectiveness and pertinence of product design schemes.
\end{abstract}

\section{Introduction}

The advancement of science has gradually made intelligent manufacturing the mainstream of the development of manufacturing industries in various countries, and computers have also provided a lot of technical support for this. For example, intelligent detection based on computer neural network can help special machinery to better realize fault diagnosis and structural optimization; data induction based on analogy reasoning can help people establish a comprehensive machinery manufacturing knowledge system; intelligent optimization based on genetic algorithm is also special indispensable technology in mechanical design. In the current research, people still need to continuously improve computer intelligence technology to better improve the rationality of mechanical structure optimization.
Abroad, research on computational intelligence and structural optimization has accumulated a lot of excellent results. Moreira tried to use genetic calculation and simulated annealing to solve the problem of structural optimization. $\mathrm{He}$ and his team studied the performance of two random search methods and applied them to the optimization of pin-connected steel structures. From the research results, genetic algorithm and simulated annealing can indeed play a very good role in structural optimization. If they can be embedded in a single parameter algorithm, better performance can be achieved through a hybrid scheme [1]. Reynolds proposed a new reverse adaptive method for automatically generating solutions to initial design and redesign problems. He carried out inverse adaptive analysis of the initial finite element problem and refined the low-stress area of the finite element mesh according to element 
subdivision. After that, he deleted all low-stress subdivision elements and repeated the process continuously. This method can effectively improve some of the shortcomings in the existing optimization scheme, but there is still a lot of room for improvement in the efficiency of optimization [2]. Motta and his team developed an efficient calculation tool for robust structural optimization. Due to the combination of multiobjective optimization, normal boundary intersection, and normalized normal constraints, this integrated tool can effectively obtain the best solution for robust design optimization. Of course, the tool needs more experiments to ensure the stability of its calculations [3].

Although domestic research on computer technology in structural optimization design started relatively late, it has developed very rapidly. $\mathrm{Qu}$ et al. proposed a reasoning method of fuzzy design knowledge, which can refer to the relevant detailed information of product design in a certain order in the reasoning process of product parameters. The prerequisite for this purpose is to establish a hierarchical model to express the product design, processing, and technology on the basis of meeting the product design accuracy requirements [4]. Bijuan et al. conducted research on the parameter analysis and optimization of the damping structure of the tubular transition layer of agricultural machinery. He introduced the concept of transition layer on the basis of the traditional constrained damping structure, combined with computer technology, and proposed a new type of tubular damping structure. From the experimental results, the optimized agricultural machinery can better deal with the severe vibration and impact that may occur during the work process, but the stability of the tubular structure needs to be appropriately improved [5].

Through reading materials and market research, this article understands the problems encountered in the current mechanical product design process and analyzes it. It is concluded that the application research of knowledge reuse technology has brought progress to the design and development of mechanical products and the development of manufacturing enterprises, which means using the idea of functional analysis system technology and quality function deployment technology to successfully complete the transformation between customer demand information and structural design parameters and focus on the process of using multilevel classification technology to generate design plans and discuss the basic elements of multilevel decomposition. Classification method, indexing process, and matching reasoning form the complete idea of applying knowledge reuse technology to generate design scheme.

Introduction systematically introduces the research overview of the overall structure optimization design of special machinery and expounds the current main problems in the overall structure optimization design of special machinery and the main work and research content of this article. Section 2 explains intelligent optimization design technology and method of mechanical structure and introduces structural intelligent optimization, genetic algorithm, data mining technology, ANSYS-based optimization technology, and penalty function constraints. Section 3 includes data preprocessing part, detailed description of the corresponding method of simulation experiment in this paper, and the establishment of experimental model. Section 4 mainly introduces the establishment of a special machine model based on ANSYS and intelligence and conducts related experiments to optimize the implementation strategy. Section 5, Summary and Prospects, summarizes the work done in this article, states the innovation and effectiveness of the method proposed in this article, and proposes the next research direction.

\section{Technology and Method of Intelligent Optimal Design of Mechanical Structure}

2.1. Structural Intelligent Optimization. The intelligent optimization design of the structure is essentially an optimization process; that is, a solution is determined in the solution space of the mechanical design to make the objective function obtain the minimum value under the condition of satisfying the state variable constraints [6]. The more complex the mechanical design is, the more constraints and variables it contains. Not only that, but some constraints are even discrete and difficult to describe quantitatively. In the process of structural optimization, the final evaluation standard for the quality of the design plan will be reflected in the way of evaluation function [7]. If there is only a single optimization goal, then the optimization process will be relatively simple. But in actual work, there may be two or more optimization goals that have contradictory relationships with each other. At this time, it is necessary to integrate multiple methods such as multiobjective fuzzy optimization, evaluation function method, and goal planning method to optimize the overall structure [8].

Traditional optimization algorithms include mathematical programming method, optimal criterion method, and so on. These classic optimization methods are limited by various conditions, so they can be used in a limited range, and it is difficult to meet the optimization of special machinery structures in complex environments [9]. For this reason, it is necessary to introduce computer technology into intelligent optimization methods, imitate the evolution and development process of nature, and combine logic, mathematics, and science to realize project design and optimization. In the process of searching for the optimal solution, the most representative one is genetic algorithm (GA). In addition, simulated annealing (SA), fuzzy systems, and artificial neural networks (ANN) are common search optimization techniques at this stage $[10,11]$.

2.2. Genetic Algorithm. The essence of genetic algorithm is to simulate the laws of biological heredity and evolution in the natural environment and obtain a highly adaptive algorithm that can search for global probability [12]. The genetic algorithm strictly follows the natural law of survival of the fittest in the calculation process. In each genetic process, only the group with effective information can iterate to the next round. After multiple iterations of calculation, the operations of selection, crossover, and mutation are continuously performed on individuals, until the optimal solution that satisfies the conditions is obtained [13]. 
2.2.1. Basic Genetic Algorithm. The most basic genetic algorithm operation process must first clarify the objective function, variable, variable search range, and search precision of the problem, then determine the length of each design variable code, and then code the variable [14]. In the process of population initialization, an initial population containing a certain number of individuals can be randomly generated according to the selected coding method; namely,

$$
\operatorname{popi}(t), t=1,2,3, \ldots, n \text {. }
$$

Here, $t$ represents the initial population number, which is used to evaluate and select the fitness value of individuals to complete genetic processes such as crossover and mutation. It can reflect the pros and cons of all individuals in the form of a function, and its expression satisfies

$$
f i=\text { fitness }(\operatorname{pop} i(t)),
$$

where $f_{i}$ means to select highly adaptable individuals from the group to form a new group. In the genetic process, the process of selection can also be seen as a process of replication, that is, selecting excellent individuals with strong adaptability from the population and forming them into a new population [15]. It is necessary to comprehensively consider the fitness value when selecting operators. Normally, the calculated selection probability of each individual satisfies the formula

$$
P i \frac{f i}{\sum_{i=1}^{n} f i} .
$$

Combining formula (3), when selecting outstanding individuals from the current group popi $(t)$ to inherit to the next generation, the new group constituted satisfies

$$
\operatorname{newpop}(t+1)=\{\operatorname{pop} q(t), q=1,2,3, \ldots, n\},
$$

where newpop $(t+1)$ represents the probability obtained by the crossover method. The crossover process refers to randomly selecting two parents of individuals, and then according to the determined crossover method and probability, part of the individual genes of the parents are crossed to form two brand new offspring individuals [16]. Generally speaking, there are one-point intersection, two-point intersection, and multipoint intersection.

The process of mutation is to reverse the value of a certain gene in the chromosome. Generally speaking, it is to call 0 and 1 in the binary string. If the mutated parent individual is [01100110], when the fourth point is mutated, then the mutated offspring individual is [01110110]. The main role of mutation operator in the genetic process is to enhance the diversity of individuals in the entire population. The more significant the differences between individuals, the lower the probability of local optimization [17].

2.2.2. Improved Genetic Algorithm. The basic genetic algorithm can solve most simple optimization problems, but there are still some shortcomings in the face of complex multiobjective optimization. In order to meet the requirement of designing and optimizing the overall structure of special machinery in this paper, genetic algorithm needs to be improved appropriately. Generally speaking, the improvement methods of genetic algorithms are mainly divided into improved coding methods, adding advanced operators, and combining other search algorithms.

Binary encoding is one of the commonly used encoding methods in genetic algorithms. Suppose ceil represents rounding to positive infinity, perk represents coding accuracy, and [low, up] represents the search range of variables under restricted conditions; then the length calculation formula satisfies

$$
l=\operatorname{ceil}\left[\log _{2}\left(\frac{\mathrm{up}-\mathrm{low}}{\text { perk }}+1\right)+1\right] .
$$
formula

The actual precision perk' of the string satisfies the

$$
\text { perk }^{\prime}=\frac{\text { up }- \text { low }}{2^{l}-1} .
$$

On the whole, binary coding is simple to operate and has better global search capabilities, but due to the mapping error of continuous function discretization, it cannot directly reflect the structural characteristics of the problem [18]. In order to improve the search efficiency of genetic algorithm, this paper improves it to a segmented coding method. The characteristic of segmented coding is to use two different coding schemes to divide the algorithm into preliminary search and final search. In the early stage of calculation, the genetic algorithm can perform a rough search of the whole world and first confirm the possible range of the optimal solution. In the later stage, gray coding is used to perform a more detailed search to achieve precise search in a small area.

2.2.3. Advantages of Genetic Algorithm. Compared with traditional methods, the superiority of genetic algorithms is mainly manifested in first, and under the action of genetic operators, genetic algorithms have strong search capabilities and can find the global optimal solution of the problem with a large probability. The inherent parallelism can effectively handle large-scale optimization problems. Genetic algorithm has a good global search ability, can quickly search out all solutions in the solution space without falling into the trap of rapid decline of local optimal solutions; and using its inherent parallelism, it can easily perform distributed computing to speed up the solution. However, the local search ability of genetic algorithm is poor, which makes the pure genetic algorithm more time-consuming and the search efficiency is lower in the later stage of evolution. In practical applications, genetic algorithms are prone to premature convergence. Which selection method should be adopted to keep good individuals and maintain the diversity of the group has always been a difficult problem in genetic algorithms.

2.3. Data Mining Technology. The basis of data mining is realized on the basis of metadata definition, modeling, and multidimensional data modeling. Its essence is to mine 
useful data from a large amount of data according to its related laws [19]. Simply put, it is to find the characteristics of the data through statistics and machine learning on the basis of data samples and use icons to describe them and establish their knowledge description model. With the help of models, people can dig out the value and relevance behind the information.

Data mining technology can be very helpful to the optimization design of the structure. In actual work, data can be classified, summarized, and clustered according to optimization objects and constraints and finally confirmed its association rules [20]. In the machinery manufacturing industry, data mining technology is often used for fault diagnosis of parts and components, and resource optimization is achieved by analyzing the production process.

\subsection{Optimization Technology Based on ANSYS. ANSYS is a} finite element analysis software, which is often used in computer engineering aids to solve various linear and nonlinear problems [21]. ANSYS not only has an excellent modeling level but also can effectively achieve problem solving, nonlinear analysis, and system optimization. Using ANSYS to optimize design can help users determine the optimal design plan, use ANSYS's topology optimization module to optimize the shape of the model, or confirm the best distribution of materials [22].

The optimization analysis technology based on ANSYS is usually divided into three categories; the most common is the optimization technology based on parametric design language. This optimization method can not only effectively form finite element modeling but also further realize parametric analysis and solution, so it can play a very good effect in the secondary development and optimization design of mechanical structure.

2.5. Penalty Function Constraint. Optimization problems are usually accompanied by certain constraints. The penalty function method is a constraint processing method that is often used in the solution of constraint optimization problems using intelligent algorithms [23]. As an indirect processing method, the penalty function will form a new function with the constraint term together with the objective function after imposing a certain penalty on the constraint function. Commonly used penalty functions include external point penalty function, differentiable penalty function, and multiplier method, which can also be directly divided into internal penalty function method and external penalty function method [24].

The operating idea of the external point penalty function method is to define the constraints outside the feasible region through the penalty effect, and, in the search process, it gradually moves from the outside of the feasible region to the boundary until the optimal solution that meets the requirements is found. The basic idea of the differentiable penalty function method is to approximate a nondifferentiable precise penalty function through a differentiable function. The basic idea of the multiplier method is to introduce some undetermined coefficients into the constraint function to form a new unconstrained augmentation objective function with the original objective function and finally make unconstrained augmentation by continuously modifying the multiplier vector of the constraint function. The optimal solution of the wide objective function is equal to the optimal solution of the original problem [25]. Because the parameter model studied in this paper is relatively complicated, when optimizing the parameter structure, this paper uses the advantages of computers in data storage and analysis, combined with the use of historical adaptive DE, and improves the intelligence of optimization [26].

\section{Optimization Experiment of Mechanical Structure Based on Computer Technology}

3.1. Experimental Background. Although special machinery is not manufactured and used as frequently as daily necessities, it is an essential part of the national development strategy. As a high-level weapon machine, the structural optimization and design process of artillery will inevitably involve a lot of optimization goals and constraints. This includes, but is not limited to, the overall structure of the artillery, the links of the various components of the artillery system, and finite element dynamics analysis. In order to optimize the overall structure of the artillery, it is necessary to combine genetic algorithms, data mining, ANSYS optimization, and penalty functions in computer technology.

3.2. Experimental Model Establishment. The establishment of a mathematical model of structural optimization is a very important part of using mathematical methods to solve structural optimization problems in practical engineering. In the field of special machinery manufacturing, the mathematical model of structural optimization design needs to meet the constraints of a series of equilibrium conditions to maximize the rigidity of the structure, the least flexibility, the least total weight, or the least material cost. From the perspective of abstract mathematical form, the mathematical model of structural optimization mainly includes design variables, constraint conditions, and objective functions. Towing artillery is a complicated mechanical structure. In the modeling process, it is necessary to establish a corresponding finite element model based on its structural characteristics. This article divides it into four parts: recoil part, landing part, rotating part, and fixed part.

3.3. Experiment Process. The overall idea of the experiment is as follows. First, we determine the parameter variables and their design space and establish a parameterized finite element model on this basis. Then, the parameterized model is used for finite element analysis to obtain the objective function value and the constraint value, thereby establishing the implicit correspondence between the objective function and the constraint function and the current design variables. Finally, the intelligent optimization algorithm is used to optimize the overall structure of the artillery, and the optimization results are given. 
In order to further examine the search efficiency of the improved genetic algorithm and its influence on structural optimization, this paper records the operating parameters of the basic genetic algorithm and compares them with the improved algorithm. When dealing with constrained optimization problems, this paper also compares the constraint capabilities of several common penalty functions, which reflects the excellent constraint processing capabilities of the multiplier method.

\section{Application of Computer Technology in Optimal Design of Overall Structure of Special Machinery}

\subsection{Establishment of Special Machinery Model Based on ANSYS}

\subsubsection{Application of Special Machinery Parameterized Model} in Structural Optimization. Parameterization is to use a set of design parameters to agree on the relationship of the structure size when the structure shape is basically fixed and then change the structure shape through size drive. Unlike traditional design methods, parametric design can store the entire involved process and design a family of models that are similar in shape and function instead of a single model. It is precisely because of the parametric modeling technology that the transmission of data changes between different levels has the uniqueness and immediacy. On the whole, parametric technology has a very wide application background and important significance in the design of special machinery products and systems.

When building the overall structure model of the artillery, it is not only necessary to determine the multiobjective function and constrain the design but also to select variables based on requirements. Table 1 shows the variable ranges of various variables in the structural model. It can be seen from Table 1 that when designing variable spaces, the results of historical data analysis give priority variables as much space as possible. For nonpriority variables, you can refer to the priority basic model and give it a data fluctuation range of about $30 \%$. Among them, the data of M8 and M9 will directly affect the situation of the gun tail colliding with the ground when the firing angle range is large. It is impossible to judge whether the structure itself interferes. Therefore, the interference check function must be added when the new group is optimized; that is, the maximum between recoil displacement and M8 and M9 is a constraint that the new group must meet.

After selecting the optimized variables based on historical data, this paper analyzes the sensitivity of each parameter to the stability of the overall structure, the weight of the whole gun, the stress of the frame, and the muzzle disturbance. Figure 1 is a statistical graph of parameter sensitivity of the overall structure of the model.

It can be seen from Figure 1 that the parameters that have a greater impact on the stability of the model structure are M2, M3, M4, M7, and M14. The parameters that have a greater impact on muzzle disturbance are M3, M4, and M14. When M3 increases by $10 \%$, the muzzle disturbance of the
TABLE 1: Variable range of various variables in structural model.

\begin{tabular}{lccccccc}
\hline Design variable & M1 & M2 & M3 & M4 & M5 & M6 & M7 \\
\hline Design upper limit & 125 & 350 & 5200 & 650 & 1400 & 600 & 1000 \\
Design lower limit & 0 & 150 & 2000 & 200 & 1100 & 400 & 500 \\
Design variable & M8 & M9 & M10 & M11 & M12 & M13 & M14 \\
Design upper limit & 1200 & 1200 & 460 & 550 & 8 & 8 & 680 \\
Design lower limit & 600 & -400 & 200 & 350 & 2 & 2 & 420 \\
\hline
\end{tabular}

artillery increases from 2780 to 3227 , and the rate of disturbance increases as high as $16.08 \%$. The parameters that have a greater impact on the total mass of the artillery are M3, M10, and M12. It can be seen that in the optimization, the upper and lower limits of the above parameters should be given sufficient width, and the interference of the structure should be considered, and the interference check of the final feasible design plan should be done.

4.1.2. Intelligently Optimized Implementation Strategy. In the process of structural optimization, the specific optimization steps are as follows. (1) Extract valuable data from past models to form a source database and perform equivalent processing on all parameter data in it to eliminate structural differences caused by performance differences. (2) Determine the variable range of all parameters and ensure that each parameter will not interfere or conflict with each other. At the same time, confirm the structural design variables and various constraints. (3) Form an optimized mathematical model and determine the objective function and penalty function as the basic model of genetic algorithm. (4) Parameterize the finite element model, encode the chromosome, analyze the finite element of different design parameters, and confirm the correspondence between the various data in the structure. (5) Complete genetic calculation through selection, crossover, and mutation operations and find the optimal solution through genetic algorithm to achieve system optimization. Figure 2 shows the changes of various parameters of the model after intelligent optimization.

It can be seen from Figure 2 that the optimized overall structure has a certain change from the beginning. For example, the height of the live wire is reduced from $900 \mathrm{~mm}$ to $600 \mathrm{~mm}$, the length of the frame is changed from 3600 to 3000 , and the wall thickness of the frame is changed from 5 to 3. After further understanding the dynamic performance curve of the optimized model, it can be determined that the dynamic performance of the model meets the requirements of use, that is, meets the requirements of accuracy and stability. Through subsequent optimization and adjustment of parts, the final optimization plan can be realized.

\subsection{Realization of Mechanical Structure Optimization Based on Genetic Algorithm}

\subsubsection{Improved Genetic Algorithm and ANSYS Collaborative} Research. In actual work, it is difficult to establish an accurate mathematical model for large complex structures. Therefore, special finite element analysis tools are required for analysis and calculation. Improved genetic algorithm and 


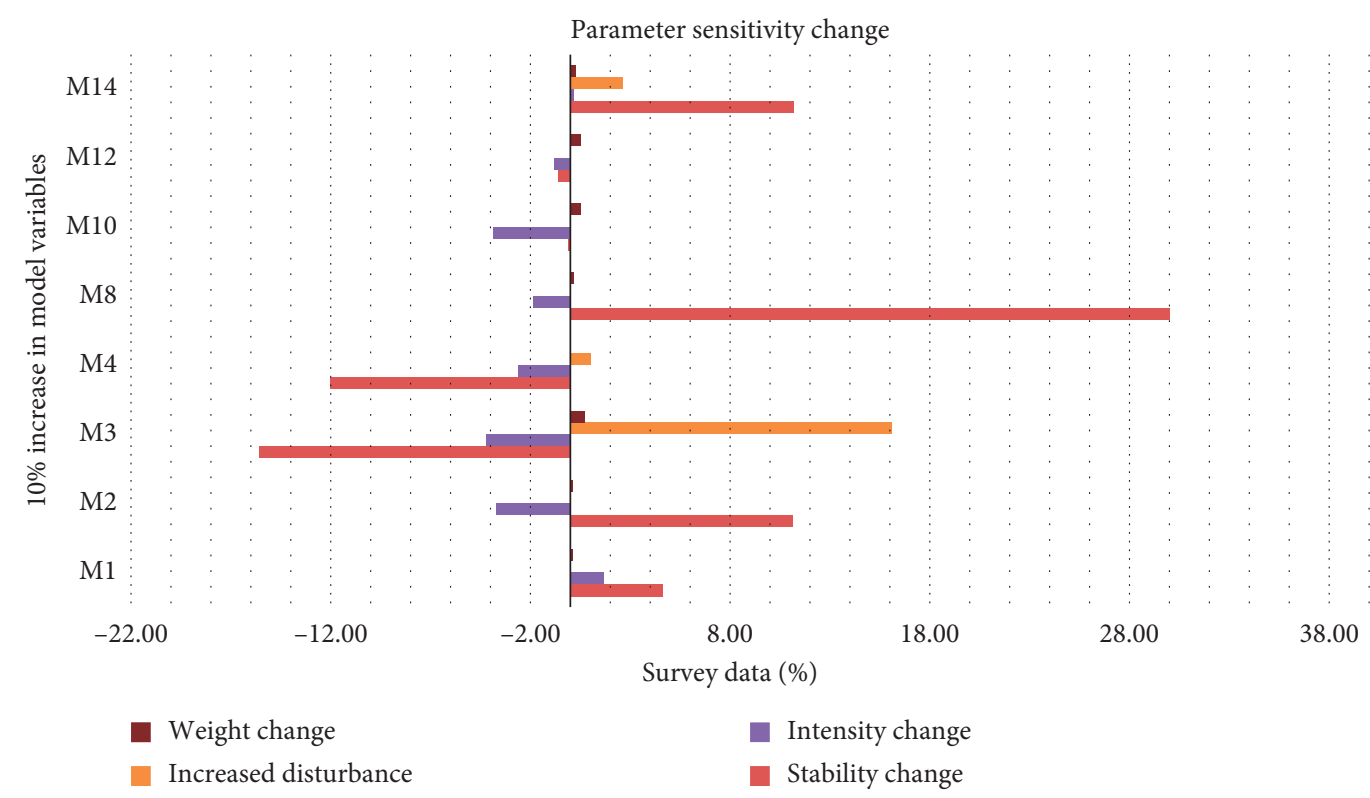

FIgURE 1: Parameter sensitivity statistical graph of the overall structure of the model.

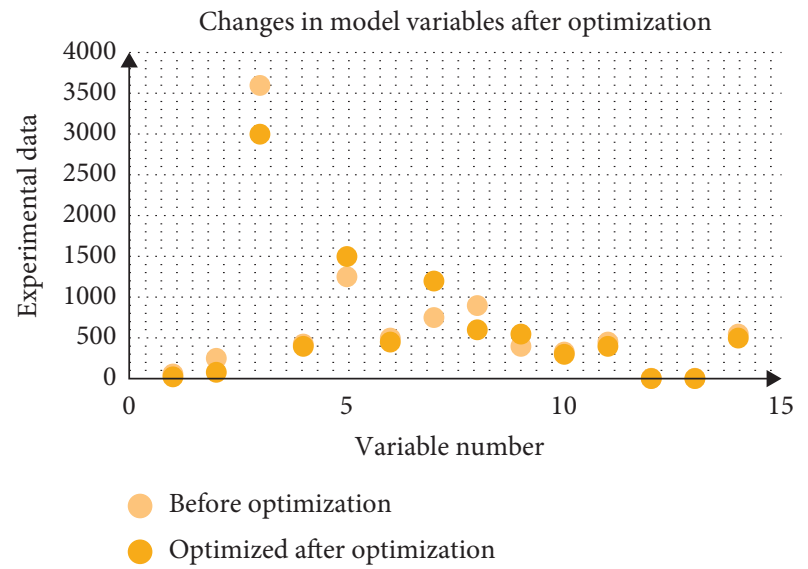

FIGURE 2: Changes in various parameters of the model after intelligent optimization.

ANSYS for collaborative calculation can not only effectively ensure the correct rate of calculation but also reduce some difficulties that may be encountered in the programming process and more efficiently achieve structural optimization design. Figure 3 is a comparison diagram of optimization results between the basic genetic algorithm and the improved genetic algorithm. Among them, A1-A5 represent the number of population sizes in five test functions, and $\mathrm{B} 1-\mathrm{B} 5$ represent the number of optimal solutions obtained by five test functions in 100 optimization calculations.

It can be seen from Figure 3 that when solving simple optimization problems such as Project 1 and Project 3, the basic genetic algorithm can also search for the optimal solution with a greater probability. But when faced with a highly discrete multiobjective optimization problem, it is difficult to determine the optimal solution in the global search of the basic genetic algorithm. And for the improved genetic algorithm, even in the face of complex optimization tasks, the probability of obtaining the optimal solution can be maintained above $90 \%$. After further understanding the optimization, it can be found that for simple items 1 and 3, there is no obvious difference in the calculation time before and after the genetic algorithm improvement. However, as the calculation difficulty increases, the efficiency improvement of the improved genetic algorithm will become more obvious. In Project 5, the basic genetic algorithm took 328.64 seconds, while the improved time was only 86.31 seconds, which increased the efficiency by $73.74 \%$. Overall, the improved genetic algorithm has better robustness and can find the optimal solution in a shorter calculation time. For complex problems such as the optimization of the overall structure of special machinery, the improved genetic algorithm can obviously greatly help improve the optimization efficiency.

4.2.2. Modeling Stability Analysis Based on Penalty Function. In the process of using genetic algorithm to optimize the overall structure of special machinery, more variables and constraints will appear. For this reason, it is necessary to introduce a penalty function as a constraint function to ensure that the genetic algorithm can better achieve system optimization. Figure 4 is a comparison of three different forms of penalty function constraints. Among them, M1-M4 represent the number of times that the constraint conditions are met when the four types of test functions are calculated 100 times. N1-N4 represent the number of times the optimal solution is obtained when the four types of test functions are calculated 100 times.

It can be seen from Figure 4 that in a total of 400 optimization calculations, the three penalty functions meet the constraint conditions relatively well, and the multiplier method still has a slight advantage in comparison. However, when comparing the number of times to obtain the optimal solution, 


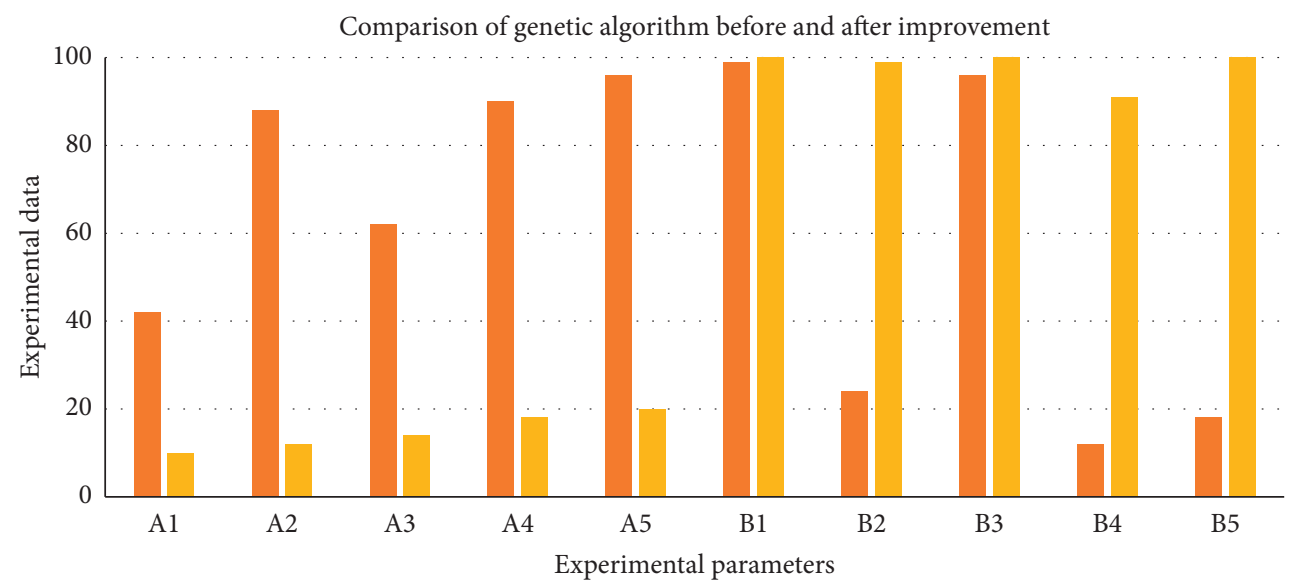

Basic genetic algorithm

- Improved genetic algorithm

FIgURE 3: Comparison chart of optimization results of basic genetic algorithm and improved genetic algorithm.

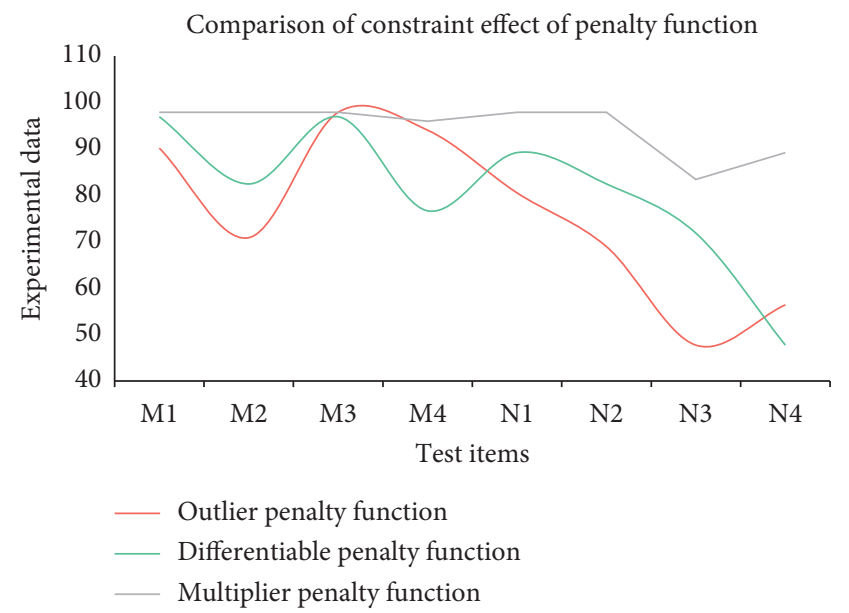

Figure 4: Comparison of constraint effects of three different penalty functions.

it can be found that the multiplier method has significantly better performance than the exterior point penalty function and the differentiable penalty function. On the whole, using the multiplier method to constrain the overall structure of special machinery can achieve better results.

4.3. Finite Element Analysis Based on Ansys. APDL parametric modeling is used to establish a finite element analysis model from bottom to top. Its mechanical behavior is based on the principle of no pressure, and only the pulling force is considered. The anchor rod and the raft are connected by a common node to ensure their deformation coordination under load. The specific results are shown in Table 2.

From Figure 5, we can see that the fitness change is very small after the 150th generation of evolution, and there is basically no change in fitness after the 250th generation. The change in the weight of the whole gun changes greatly before the 120th generation. After the change is small, there is basically no change after the 250th generation. It can be seen
TABle 2: Overall structure weight change data sheet.

\begin{tabular}{lccccccc}
\hline Number of evolutions & 0 & 50 & 100 & 150 & 200 & 250 & 300 \\
\hline$A$ & 2.90 & 3.41 & 3.84 & 4.41 & 4.25 & 4.59 & 4.71 \\
$B$ & 2.92 & 3.56 & 4.20 & 4.01 & 4.33 & 4.52 & 4.53 \\
$C$ & 2.85 & 3.31 & 4.13 & 4.45 & 4.27 & 4.59 & 4.51 \\
$D$ & 3.40 & 3.73 & 3.93 & 4.19 & 4.46 & 4.77 & 4.75 \\
$E$ & 3.29 & 3.53 & 3.96 & 4.08 & 4.43 & 4.59 & 4.71 \\
\hline
\end{tabular}

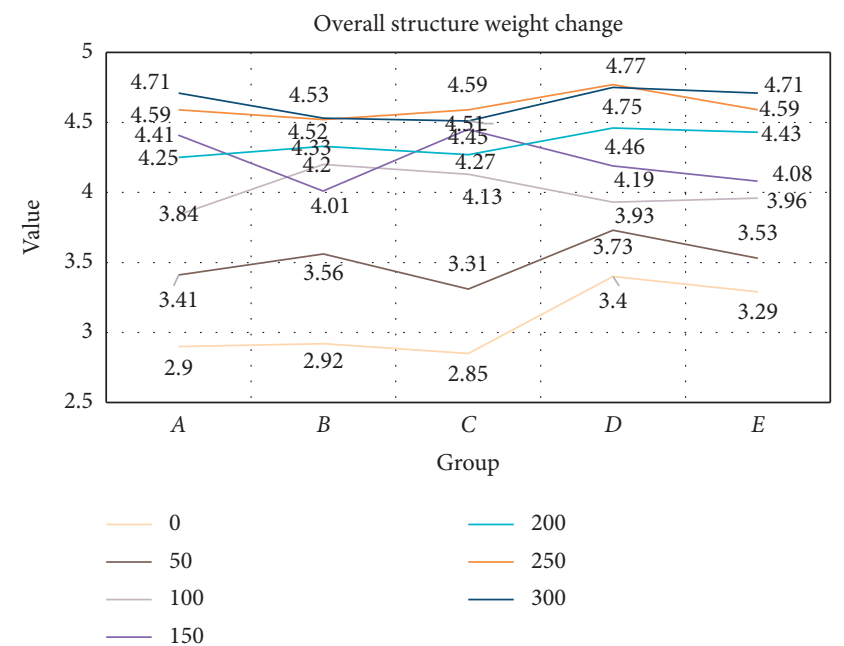

FIGURE 5: Analysis chart of overall structure weight change.

from the above trends that the evolution 300 has made the optimization mature, and the optimization results have basically reached the optimal value. The optimization effect of increasing the evolution algebra is not great.

\section{Conclusions}

This paper analyzes the special machinery model based on ANSYS. In this paper, a mathematical model is established around the overall structure of the artillery. In the determination of multiple objective functions and constraint 
design, genetic algorithms are introduced to improve various problems in the optimization process. When confirming the optimized variables and variable ranges, a data fluctuation range of about $30 \%$ was given to nonfinite variables. After understanding the parameter sensitivity of the overall structure of the artillery, the parameters that have a greater impact on performance were optimized. Because the artillery parameter model is more complicated, when optimizing the parameter structure, this article uses the advantages of the computer in data storage and analysis, combined with the past historical data to improve the intelligence of the optimization. In this paper, the overall optimization design of the artillery model is carried out according to the scientific and intelligent optimization steps, and the random election method is used to ensure the diversity of the population in the genetic process. From the experimental results, the adjustment of various parameters in the model makes the model more in line with dynamic performance in terms of accuracy and stability.

In this paper, the optimal design of mechanical structure is realized based on genetic algorithm. As a finite element analysis software, the combination of ANSYS and genetic algorithm can effectively promote the overall structure optimization of special machinery. In the experiment, this paper found that the basic genetic algorithm can solve most simple objective optimization tasks, but there are still many shortcomings in the face of multiobjective tasks with high discrete variables. Because this paper has improved the genetic algorithm, from the experimental data, the improved genetic algorithm can greatly improve the optimization calculation efficiency on the basis of ensuring the optimization accuracy and has higher robustness. This paper compares the constraint performance of the three types of penalty functions: external point penalty function, differentiable penalty function, and multiplier method. From the final result, the multiplier method has better performance in two types of tests: satisfying the constraints and obtaining the optimal solution.

With the advent of the era of automation, how companies can keep up with the rapid development of the market and correctly grasp the direction of product development and design is the key to winning this opportunity. Through the study of knowledge reuse, this paper has realized the purpose of shortening the development cycle and satisfying the needs of customers to the greatest extent. It proposes a method to realize the scientific analysis of market demand information through the integration of quality function deployment technology and functional system analysis technology. The transformation of demand information and functional parameter design and a method for calculating transformation weights are proposed, which provide important weight coefficients for the product instance matching stage. This paper uses multilevel example decomposition to achieve the decomposition of the target design scheme and uses the subspace algorithm to classify the existing design examples. And it is proposed to complete the calculation process of matching the target design scheme with the existing design examples.

\section{Data Availability}

The data used to support the findings of this study are available from the corresponding author upon reasonable request.

\section{Conflicts of Interest}

The authors declare that they have no conflicts of interest.

\section{References}

[1] H. S. Moreira, J. Lucas de Souza Silva, M. V. Gomes dos Reis, D. de Bastos Mesquita, B. H. Kikumoto de Paula, and M. G. Villalva, "Experimental comparative study of photovoltaic models for uniform and partially shading conditions," Renewable Energy, vol. 164, no. 3, pp. 58-73, 2021.

[2] D. Reynolds, J. Mcconnachie, P. Bettess et al., "Reverse adaptivity-a new evolutionary tool for structural optimization," International Journal for Numerical Methods in Engineering, vol. 45, no. 5, pp. 529-552, 2015.

[3] R. D. S. Motta, S. M. B. Afonso, P. R. Lyra, and R. B. Willmersdorf, "Development of a computational efficient tool for robust structural optimization," Engineering Computations, vol. 32, no. 2, pp. 258-288, 2015.

[4] X. Qu, G. Xu, X. Fan, and X. Bi, "Intelligent optimization methods for the design of an overhead travelling crane," Chinese Journal of Mechanical Engineering, vol. 28, no. 1, pp. 187-196, 2015.

[5] Y. Bijuan, S. Dagang, Z. Wenjun et al., "Parameter analysis and optimization of tubular transitional layer damping structure for agricultural machinery," Transactions of the Chinese Society of Agricultural Engineering, vol. 31, no. 22, pp. 56-62, 2015.

[6] C. Wang, Z. G. Zhao, T. Zhang et al., "Structure optimization and its validation for compound power-split e-CVT," China Journal of Highway \& Transport, vol. 28, no. 3, pp. 117-126, 2015.

[7] H. A. Pham, "Reduction of function evaluation in differential evolution using nearest neighbor comparison," Vietnam Journal of Computer Science, vol. 2, no. 2, pp. 121-131, 2015.

[8] R. Acedo-Hernández, M. Toril, S. Luna-Ramírez, and C. Úbeda, "A PCI planning algorithm for jointly reducing reference signal collisions in LTE uplink and downlink," Computer Networks, vol. 119, pp. 112-123, 2017.

[9] L. Yang, S. Liu, S. Tsoka, and L. G. Papageorgiou, "Mathematical programming for piecewise linear regression analysis," Expert Systems with Applications, vol. 44, pp. 156-167, 2016.

[10] G. Müller and R. Brendel, "Simulated annealing of porous silicon,” Physica Status Solidi (A), vol. 182, no. 1, pp. 313-318, 2015.

[11] A. K. Jain, J. Mao, and K. M. Mohiuddin, "Artificial neural networks: a tutorial,” Computer, vol. 29, no. 3, pp. 31-44, 2015.

[12] B. A. Norman, "A genetic algorithm methodology for complex scheduling problems," Naval Research Logs, vol. 46, no. 2, pp. 199-211, 2015.

[13] G. A. Rauwolf and V. L. Coverstone-Carroll, "Near-optimal low-thrust orbit transfers generated by a genetic algorithm," Journal of Spacecraft \& Rockets, vol. 33, no. 6, pp. 859-862, 2015.

[14] G. M. Morris, D. S. Goodsell, R. S. Halliday et al., "Automated docking using a Lamarckian genetic algorithm and an 
empirical binding free energy function," Journal of Computational Chemistry, vol. 19, no. 14, pp. 1639-1662, 2015.

[15] J. A. Niesse and H. R. Mayne, "Global optimization of atomic and molecular clusters using the space-fixed modified genetic algorithm method," Journal of Computational Chemistry, vol. 18, no. 9, pp. 1233-1244, 2015.

[16] R. Tavakkoli-Moghaddam, J. Safari, and F. Sassani, "Reliability optimization of series-parallel systems with a choice of redundancy strategies using a genetic algorithm," Reliability Engineering \& System Safety, vol. 93, no. 4, pp. 550-556, 2017.

[17] R. Kumar Sahu and V. Vikas Thakare, "Optimization of symmetric linear phase low pass FIR filter using genetic algorithm," Acta Radiologica, vol. 45, no. 6, pp. 679-688, 2015.

[18] J.-P. Heo, Y. Lee, J. He, S.-F. Chang, and S.-E. Yoon, "Spherical hashing: binary code embedding with hyperspheres," IEEE Transactions on Pattern Analysis and Machine Intelligence, vol. 37, no. 11, pp. 2304-2316, 2015.

[19] D. A. Adeniyi, Z. Wei, and Y. Yongquan, "Automated web usage data mining and recommendation system using K-nearest neighbor (KNN) classification method," Applied Computing and Informatics, vol. 12, no. 1, pp. 90-108, 2016.

[20] A. Buczak and E. Guven, "A survey of data mining and machine learning methods for cyber security intrusion detection," IEEE Communications Surveys \& Tutorials, vol. 18, no. 2, pp. 1153-1176, 2017.

[21] G. Hattori and A. L. Serpa, "Contact stiffness estimation in ANSYS using simplified models and artificial neural networks," Finite Elements in Analysis and Design, vol. 97, pp. 43-53, 2015.

[22] S. Kato, T. Takagi, and M. Kawahara, "A finite element analysis of mach reflection by using the Boussinesq equation," International Journal for Numerical Methods in Fluids, vol. 28, no. 4, pp. 617-631, 2015.

[23] A. Panda and S. Pani, "A symbiotic organisms search algorithm with adaptive penalty function to solve multi-objective constrained optimization problems," Applied Soft Computing, vol. 46, no. C, pp. 344-360, 2016.

[24] M. Brzoza-Brzezina, M. Kolasa, and K. Makarski, "A penalty function approach to occasionally binding credit constraints," Economic Modelling, vol. 51, pp. 315-327, 2015.

[25] B. C. Fabien, "An extended penalty function approach to the numerical solution of constrained optimal control problems," Optimal Control Applications \& Methods, vol. 17, no. 5, pp. 341-355, 2015.

[26] Y. J. Song, D. Q. Wu, A. W. Mohamed, X. B. Zhou, B. Zhang, and W. Deng, "Enhanced success history adaptive DE for parameter optimization of photovoltaic models," Complexity, vol. 2020, Article ID 6660115, 22 pages, 2020. 